\title{
Criminologie
}

\section{Vers un paradigme des inter-relations sociales? Pour une reconstruction du champ criminologique}

\section{Alvaro Pires et Françoise Digneffe}

Volume 25, numéro 2, 1992

Nouvelles connaissances et nouvelles questions en criminologie

URI : https://id.erudit.org/iderudit/017321ar

DOI : https://doi.org/10.7202/017321ar

Aller au sommaire du numéro

Éditeur(s)

Les Presses de l'Université de Montréal

ISSN

0316-0041 (imprimé)

1492-1367 (numérique)

Découvrir la revue

Citer cet article

Pires, A. \& Digneffe, F. (1992). Vers un paradigme des inter-relations sociales? Pour une reconstruction du champ criminologique. Criminologie, 25(2), 13-47. https://doi.org/10.7202/017321ar
Résumé de l'article

One of the abiding debates in criminology contrasts social reactions to deviance with explorations of the causes in criminality. In this article, the authors note that the two paradigms consider crime in totally different ways, one as a raw fact, the other as a purely social construction. The authors propose a new paradigm which integrates at the same time contributions of sociology, clinical criminology from the School of Louvain, and feministic perspectives. This proposal differs from those advanced by left and right realists. 
VERS UN PARADIGME DES INTER-RELATIONS SOCIALES ? POUR UNE RECONSTRUCTION DU CHAMP CRIMINOLOGIQUE Alvaro P. Pirez ${ }^{1}$

Françoise Digneffe ${ }^{2}$

One of the abiding debates in criminology contrasts social reactions to deviance with explorations of the causes in criminality. In this article, the authors note that the two paradigms consider crime in totally different ways, one as a raw fact, the other as a purely social construction. The authors propose a new paradigm which integrates at the same time contributions of sociology, clinical criminology from the School of Louvain, and feministic perspectives. This proposal differs from those advanced by left and right realists.

Un des problèmes majeurs qui a affligé la criminologie depuis les années soixante est sans doute le fameux clivage entre une criminologie du passage à l'acte et une criminologie de la réaction sociale. Où en est la recherche empirique et thérique sur cette question? Peut-on dépasser cette opposition? La criminologie de la réaction sociale est-elle inutile à la criminologie clinique? Peut-on voir dans les approches féministes un apport à la construction d'un nouveau modèle? Voilà quelques-unes des questions que nous souleverons dans cet article ${ }^{3}$.

Nous sommes convaincus que le débat sur les «deux criminologies» relève d'un problème sérieux. En effet, il ne s'agit pas selon nous d'un simple casse-tête né d'un abus de langage, d'une sorte de jeu intellectuel ou encore d'un dérapage irresponsable de notre discipline. Pourtant, on a souvent choisi d'ignorer ce problème. Ceci n'empêche que la pratique de la recherche a ouvert des voies que la réflexion théorique avait ignorées d'une part et que certains auteurs ont apporté des véritables contributions théoriques à la solution de ce problème d'autre part. La conception d'ensemble de cet article s'est élaborée lors d'un séjour d'un semestre de Françoise Digneffe à l'Université d'Ottawa. Les discussions que les deux auteurs de l'article ont pu avoir à cette occasion ont permis de mettre en évidence la convergence des question-

1. Professeur, Département de criminologie, Universitê d'Ottawa, 1, Stewart, Ottawa, Ont. Canada KIN 6N5

2. Professeure, Ecole de Criminologie, Université Catholique de Louvain, 1, Place de 1'Université, 1348 Louvain-la-Neuve, Belgique

3. Nous remercions les commentaires faits a la première version de ce texte par L. Beauchenes, M. Jaccoud, P. Landreville et C. Parent, aussi bien que par un de nos appréciateurs anonymes. 
nements paradigmatiques qui se posent à partir de recherches différentes $^{4}$. D' une part, certains analystes critiques du fonctionnement de la justice pénale et des théories de la peine, etc., (Pires, 1990a ; 1991) font apparaître la nécessité d'une prise en compte des «manières de faire" (ou comportements) en relation avec la réaction pénale (ce que ne propose pas la criminologie de la réaction sociale). D'autre part, la réflexion criminologique, plus particulièrement au niveau de la clinique, telle qu'elle se poursuit à l'Université de Louvain, fait de plus en plus apparaître la nécessité d'intégrer la «manière de définir » et du dépassement des oppositions entre les «deux criminologies» (coll., 1990).

Cette nécessité résulte de considérations à la fois théoriques et éthiques ou pratiques. Théoriques en ce sens que l'impossibilité des modèles précédents à résoudre de manière satisfaisante un bon nombre de problèmes apparaît de plus en plus (Khun, 1962). Éthiques et pratiques, dans la mesure où la réflexion criminologique nous paraît devoir être guidée par un objectif de respect des droits de la personne et de reconnaissance de chacun et chacune au sein des relations sociales, pour une société émancipatrice. Cette exigence suppose un processus de communication et une prise en considération des acteurs à tous les niveaux de l'analyse et ne paraît pas pouvoir être rencontrée dans le cadre des paradigmes existants.

Dans ce travail, nous attirerons l'attention sur le fait que les deux manières actuelles de concevoir l'objet de la criminologie sont unidimentionnelles et unilatérales et que les clivages que traverse notre discipline vont au-delà de cette question paradigmatique. On mettra ensuite en évidence les contributions liées au développement de la criminologie clinique telle qu'elle est conçue par De Greeff puis Debuyst d'une part et par les études féministes sur les comportements problématiques des femmes d'autre part. Enfin, nous proposerons un modèle pour dépasser les deux paradigmes actuels tout en tenant compte des contributions précédentes. Précisons ici que le traitement de ces objectifs sera nécessairement inégal. Ceci paraît inévitable compte tenu de la complexité de ces points et des priorités que l'on s'est données.

4. Cependant, cet article a été rédigé par Alvaro Pires après le départ de Françoise Digneffe. La participation de celle-ci consiste principalement dans le texte d'une conférence faite à Ottawa en décembre 1991 sur «Quelques éléments du parcours des idées en criminologie clinique à l'École de criminologie de Louvain». Cette étude s'inscrit dans le cadre d'une recherche plus générale sur l'histoire de la criminologie qui peut se réaliser grâce à un financement du Fond National de la Recherche Scientifique belge. Elle regroupe des chercheurs belges, canadiens et français. 


\section{LES DEUX DIMENSIONS DU CRIME ${ }^{5}$}

Selon nous, la plupart des criminologues sont d'accord pour dire que le crime n'existe pas sans une règle, une loi qui nous indique - de manière approximative - qu'une telle action, et pas une autre, pourra être vue comme un crime. C'est d'ailleurs cela qui nous permet de dire que la peine de mort officiellement appliquée n'est pas un meurtre et que le prélèvement des impôts à la source n'est pas un vol ${ }^{6}$. Bien sûr, ce consensus est au fond assez limité: aussitôt établi, aussitôt défait. C'est que les criminologues ne tirent pas tous les mêmes conséquences de ce fragile point de départ ; en fait, ils finissent même par étudier des choses remarquablement divergentes.

Pour bien saisir ce qui se passe dans notre champ sur la conception de l'objet d'étude, il faut prendre acte du fait que la criminalité et une problématique qui renvoie à deux dimensions de base: d'une part, à un comportement ou à une manière de faire et d'autre part, à une qualification «criminelle» ou à une manière de définir et de réagir introduite par notre système d'organisation des droits (Debuyst, 1992b). Contrairement à ce que l'on croit, le crime n'est pas exclusivement un acte, mais le rapport entre une manière de faire (ou un conflit) et une manière institutionnelle de définir. Le mot clé pour saisir ce rapport est «devenir »: conduire vite devient une infraction lorsque cela est défini comme «excès de vitesse»; frapper devient un crime lorsque cela est défini comme "voies de fait», etc. ${ }^{7}$. Pour qu'un acte devienne crime, il faut alors que je pense à une catégorie pénale (vol, etc.) susceptible d'accueillir l'événement concerné, que je «lise » l'événement avec ces «lunettes pénales" et que je réussisse à convaincre notre système d'organisation des droits du bien-fondé de cette lecture.

Il ne suffit donc pas qu'un comportement soit dommageable, ni même très grave, pour qu'il devienne un crime. On peut même dire que la «criminalité», dans son ensemble, ne garde aucun rapport étroit avec une échelle rationnelle de gravité objective des conduites dans la société. Une manière de faire peut devenir un crime même si elle est dérisoire, et il y a des actes très graves qui ont très peu de chances de

5. Cette section reprend des remarques présentées ailleurs (Pires, 1992).

6. On se souviendra à ce propos des remarques lapidaires de Kelsen (1953, p. 46-47).

7. La notion de «devenir » a été finement proposée par Lynch et Groves (1989). Nous reprenons aussi deux de leurs exemples, mais nous ne suivons pas un bon nombre de leurs développements sur cette question. 
devenir un crime. C'est le cas des accidents de travail causant la mort à la suite d'une négligence patronale des normes de sécurité; un autre système d'organisation des droits et d'assurances prend en charge la régulation de ces illégalismes et de ces «accidents ${ }^{8}$. Il en va de même lors d'une agression physique illégale dans un jeu de hockey; encore ici, la régulation sociale et la sanction se font dans un autre cadre normatif. Un policier qui tue en service par négligence a aussi très peu de chances de devenir criminel, car ces événements ont de fortes chances d'être interprétés comme des «bavures», etc.

Nous ne voulons pas suggérer ici que la solution adéquate ou même juste à ces problèmes consiste à les « ramener à la justice pénale ", mais tout simplement attirer l'attention sur le fait que le crime n'est pas simplement une action plus ou moins dommageable ou inacceptable: il est aussi une forme de construction de la réalité.

Considérons un exemple très évocateur en langue française, l'infraction de "voies de fait", et posons-nous la question suivante: une voie de fait est-elle un fait? Pour répondre à cette question, imaginons deux scénarios. Dans le premier, un policier récemment affecté à un quartier est le témoin d'un échange de coups de poings entre deux jeunes dans la rue et dit: "Voilà une voie de fait.». En se disant cela, il définit la situation et agit comme si c'était effectivement une voie de fait. Ceci signifie qu'il intervient, amène le jeune au poste et instruit le procès pénal. Ici le conflit devient un crime. Dans le deuxième scénario, c'est un ancien policier du quartier qui connaît un ou les protagonistes en question. Il se dit: "Voilà une bagarre et se battre est une chose qu'il ne faut pas faire.». En se disant cela, il agit comme si c'était effectivement une bagarre. Il intervient également, mais il n'instruit pas une procédure pénale. Ici le conflit devient une bagarre. Or, si la voie de fait était simplement un fait capable de s'imposer de manière non équivoque, les deux policiers auraient "vu» la même chose, auraient défini la situation et agi de la même manière. Le paradoxe est qu'ils ont vu effectivement la «même" situation, mais ils n'ont pas construit mentalement et pratiquement la situation de la même façon. Et les conséquences ne sont pas non plus les mêmes. Cela veut dire que certaines agressions physiques seront étiquetées "voies de fait", d'autres non. Ceci illustre bien comment on peut venir à voir l'objet de la criminologie comme l'étude de manières de définir et de réagir à certaines situations.

8. Voir sur cette question Acosta (1988). 


\section{LES PARADIGMES DU FAIT SOCIAL ET DE LA DÉFINITION SOCIALE}

Aujourd'hui, on peut diviser grosso modo l'histoire du savoir sur la criminalité en deux grands blocs: $a$ ) ceux qui l'ont conçue quasi exclusivement comme un fait social et l'ont étudiée comme une manière de faire (ou d'être); et $b$ ) ceux qui l'ont conçue quasi exclusivement comme une définition sociale et l'ont étudiée comme une manière d'étiqueter certaines situations-problèmes et de réagir à leur égard. Bref, on a alors parlé de la criminalité tantôt comme si elle était un fait brut, tantôt comme si elle était une définition. Nous appellerons le premier bloc le paradigme du fait social, et le deuxième le paradigme de la définition sociale ${ }^{9}$.

Dans le passé, on a appelé le premier, paradigme de «criminologie étiologique» ou «du passage à l'acte». Cependant, ces termes sont imprécis parce qu'ils ne comprennent pas les recherches purement descriptives qui ont pourtant considéré le crime comme un fait social. Ils sont aussi ambigus parce que l'on considère souvent les expressions «criminologie étiologique » et «criminologie positiviste» comme synonymes alors qu'elles ne le sont pas. En effet, il est possible d'expliquer la genèse d'un événement de manière différente de celle de la criminologie positiviste ${ }^{10}$. Il y a donc différentes formes d'étiologie, et les objections les plus importantes s'adressent à la criminologie positiviste avec sa recherche d' «universaux ${ }^{11}$ » et non à l'étiologie tout court.

Le paradigme du fait social est le plus ancien et débute avec la naissance de la criminologie au XIX ${ }^{\mathrm{e}}$ siècle. En général, les recherches qui y sont attachées conçoivent le crime comme un fait social consistant plutôt qu'une réalité construite par les interactions sociales et par la

9. Le terme paradigme est employé ici pour désigner plutôt un rapport à l'objet qu'un rapport aux theories (Pires, 1983, p. 22-23). Les termes «fait social» et «definition sociale» ont été empruntés à Delruelle-Vosswinkel (1981), mais nous en avons fait une utilisation différente. L'expression «paradigme du fait social» est un peu ambiguë puisqu'on peut concevoir le fait social, à l'instar de Garfinkel, comme un accomplissement pratique ou un fait construit et non comme une chose (voir Coulon, 1987). En empruntant un terme accrédité par Lyotard $(1979$, p. 35$)$, il faut noter que cette expression désigne ici une vue «chosiste» de la réalité sociale.

10. Cette ambiguîté se trouve déjà chez Matza (1964, p. 3-12) dans la mesure où il sernble identifier l'expression «théories étiologiques» avec l'orientation de l'École positiviste centrée sur la recherche d'une différence entre les délinquants et les non-délinquants et sur le déterminisme.

11. L'expression «recherche d'universaux» par opposition aux analyses valorisant le particulier a tété appliquée à cette problématique par Laplante (1992). 
loi pénale. On étudie alors, si l'on peut ainsi dire, une seule dimension du crime: la manière de faire. On laisse alors de côté l'étude de la manière de définir et de réagir. D'une part, le crime apparaît comme une chose, une substance ayant un contour naturel et qui est évident à l'esprit. D'autre part, la loi et le système pénal sont envisagés comme étant «extérieurs», "au-dessus» et "contre» la criminalité, une sorte de miroir relativement fidèle de la nature des choses, d'appendicereflet-réaction de la réalité sociale (Pires et Landreville, 1985, p. 105). Dès lors, ces recherches donnent à la criminalité un statut d'évidence (Pires, 1979). On ne se rend pas compte du fait que la dimension juridico-pénale joue ici un rôle constitutif important (Sbriccoli, 1991). En paraphrasant Veyne $(1971$, p. 39), tout se passe comme si la justice pénale jouait pour nous le rôle du bon cuisinier du Phèdre: elle découperait les différentes situations conflictuelles selon leurs articulations véritables, en séparant la «véritable» criminalité du reste.

Déterminées en bonne partie par l' «esprit du temps», ces recherches veulent décrire les tendances statistiques de la criminalité (e.g., Quetelet), trouver les traits du crime chez l'individu (positivisme criminologique), ou encore comprendre les processus qui conduisent à la déviance ou au passage à l'acte (École de Chicago et École de Louvain). Les grandes questions de recherche sont: comment varie le taux de criminalité et quels sont ses rapports avec d'autres variables (Quetelet)?; qui est le délinquant et comment peut-on le distinguer des honnêtes citoyens (positivisme)?; comment devient-on déviant au cours d'un processus normal d'adaptation sociale et personnelle aux conditions de vie? (École de Chicago, De Greeff). La criminalité est vue tantôt comme un phénomène de masse, tantôt comme une forme de pathologie individuelle, tantôt encore comme le sous-produit d'un processus d'adaptation à des (mauvaises) conditions de vie (Pires, 1992).

Le paradigme de la définition sociale est le plus récent et se développe d'abord aux États-Unis au début des années soixante. Il a reçu aussi plusieurs appellations: théorie de l'étiquetage, criminologie de la réaction sociale, du contrôle social, constructiviste, etc. Ce paradigme se distingue du précédent par le fait de concevoir autrement l'objet. On étudie ici la manière de définir les événements et d'y réagir. Les grandes questions de recherche sont les suivantes: qui définit le comportement de qui comme déviant, criminel, etc. ? Quelles sont les conséquences de ce processus d'étiquetage? Comment se sont créées et ont évolué historiquement certaines pratiques sociales d'étiquetage? Pour ce paradigme, le monde social apparaît moins comme donné que construit par l'histoire et par nos actions actuelles (Berger et 
Luckmann, 1966). On essaie de voir comment les gens perçoivent, décrivent, interprètent et proposent des définitions de la situation et comment les gens agissent en fonction de ces définitions. Les définitions sont vues comme le résultat d'un processus historique et social. Dans cette perspective, ce qui intéresse le chercheur est l'étude de comment on en vient à étiqueter d'autres gens comme déviants, fous, délinquants, etc.

La déviance n'est plus considérée ni comme une caractéristique ni comme un comportement de la personne (Coulon, 1987, p. 13), mais comme le résultat de l'application d'une règle déterminée à une situationproblème. Dans cette perspective, la déviance serait le "produit» des agences de contrôle social. Hypersensibles à la question de la stigmatisation, ces théoriciens en sont venus à mettre en doute la possibilité même d'expliquer ou de comprendre la genèse des situationsproblèmes. En dépit de quelques exceptions, ils passent donc relativement sous silence cette question.

Un des grands mérites du paradigme de la définition sociale est d'avoir inversé la vapeur dans l'étude de la déviance et de nous avoir appris à regarder par l'autre bout de la lorgnette. Le système pénal n'est plus vu seulement comme une solution au problème de la déviance, mais comme créant et faisant partie de ce problème, car les différentes manières de définir et de réagir ont des conséquences différentes dans la suite du processus et sur la vie des gens.

\section{L'UNILATÉRALITÉ ET L'UNIDIMENSIONNALITÉ DES DEUX PARADIGMES}

Aujourd'hui, les deux paradigmes coexistent, trop souvent sous la forme d'un parfait monologue. Or, ces deux paradigmes ont une vision morcelée, unilatérale et unidimensionnelle par rapport aux différentes dimensions du problème. La question de l'unilateralite - one-sided criminology - a été soulevée par quelques études majeures (Taylor, Young et Walton, 1973, p. 165; Robert, 1973, p. 467; Debuyst, 1973, p. 290; Baratta, 1975, p. 44), et s'appliquait alors clairement aux deux paradigmes. Cependant, dans un bon nombre de cas, elle a été soulevée lorsqu' on critiquait le paradigme de la définition. Le résultat est que l'unilatéralité a été associée par la suite davantage au dernier qu'aux deux paradigmes à la fois.

Afin de mieux rendre compte de l'unilatéralité de chacun de ces paradigmes, nous présentons un tableau avec les principales critiques 
leur étant adressées sur le point qui nous concerne. Nous les avons formulées de manière à leur donner la forme d'une correspondance logique entre les côtés gauche et droit du tableau. La symétrie est donc sans doute un peu forcée, mais elle convient à nos objectifs. Le mot «indifférence» doit donc être pris avec prudence, puisque son but ici est d'égaliser le langage et de marquer l'orientation générale et minimale des deux paradigmes. Ainsi, certains auteurs ne seront pas « indifférents» à la modération des peines mais carrément contre. En outre, l'abolitionnisme, tel qu'il est soutenu entre autres par Hulsman (1982), Sheerer $(1986)$ et Haan $(1990 ; 1992)$, n'a jamais été indifférent aux politiques sociales et à la relation d'aide, bien qu'il soit relié au paradigme de la définition sociale. De même, nombre de réformateurs s'inscrivant dans le paradigme du fait social ont été très favorables à la modération des peines et ont, par exemple, lutté contre la peine de mort et en faveur des mesures communautaires ( $\mathrm{y}$ compris la probation) (Pires, 1991a; 1992).

\section{LES CRITIQUES AUX PARADIGMES}

DU FAIT SOCIAL

1. Indifférence a l'égard de la manière 1. de définir et de sélectionner la "clientele».

2. Indifférence à l'égard des conséquences négatives de la réaction pénale (rapports de pouvoir entre l'État d'une part et l'infracteur et la victime d'autre part).

3. Indifférences à l'égard de la modération, de la médiation, de la dépénalisation, etc., en matière d'intervention juridique.
DE LA DÉFINITION SOCIALE

Indifférence à l'égard de la genèse des situations-problèmes (manière de faire).

2. Indifférence à l'égard des conséquences négatives de la manière de faire (rapports de pouvoir entre les individus ou les groupes).

3. Indifférence à l'égard des politiques sociales et des relations d'aide visant à améliorer les conditions de vie.

Nous sommes persuadés que le lecteur pourra faire la part des choses si ces distinctions lui paraissent trop nettes; nous sommes bien conscients que dans la pratique peu de théoriciens ont gardé une cohérence irréprochable à ces distinctions. Mais ce n'est pas le point ici. En effet, il paraît essentiel de distinguer aussi nettement et clairement que possible les points d'éclairage et d'ombre de chacun de ces deux paradigmes afin de mieux visualiser leur partialité et leur unidimensionnalité.

On peut donc voir que la zone d'éclairage d'un paradigme correspond grossièrement à la zone d'ombrage de l'autre et que les partis pris 
de l'un s'affirment par le refus de tenir compte du point de vue opposé. Chaque paradigme a d'ailleurs développé ses propres procédures et arguments d'évitement, soit un ensemble de justifications pour eviter d'avoir à reconnaitre l'autre dimension ou l'autre point de vue. Notre objectif n'est pas d'indiquer lequel de ces paradigmes provoque les conséquences les plus regrettables ${ }^{12}$. Qu'il suffise ici de remarquer que le paradigme du fait social s'intéresse à la description ou à la genèse phénoménale de l'événement, mais laisse dans l'ombre la manière de le définir et ses effets. En revanche, le paradigme de la définition porte sur cette dimension oubliée, mais néglige la dimension «factuelle» du conflit. Or, ce «quelque chose» qu'on définit d'une certaine façon et contre lequel on réagit de différentes manières a quand même une dimension phénoménale susceptible d'être décrite et expliquée. Ainsi, la «voie de fait» et la "bagarre" sont sans doute des formes assez différentes de définition de la situation, mais l'agression physique ${ }^{13}$ demeure constante et implicite dans les deux définitions et peut être objet d'étude. Comme nous l'avons dit ailleurs, en paraphrasant encore Veyne, il est sans doute possible de trouver des mots pour décrire le banditisme à Chicago, les différentes formes de violence à l'égard des femmes, les vols de voiture à Naples, la violence de la police à Rio, le désordre causé par les fêtards à Hull, les fraudes douanières, les transactions illégales des grandes corporations, etc. Ceci n'empêche que les constructivistes ont en même temps raison lorsqu'ils remarquent que la criminalité est une forme de construction sociale de la réalité et qu'il ne faut pas parler d'elle comme s'il existait une invariante entre ces phénomènes complexes et comme si la peine, l'exclusion et la stigmatisation en étaient la panacée. Il faut done surmonter les écueils des deux paradigmes.

12. Cet exercice serait d'ailleurs futile à ce niveau de généralité, parce que la diversité interne du paradigme du fait social est énorme. Ainsi, il n'y a pas de différence remarquable d'un point de vue humaniste et libéral entre la première phase (fait social) et la deuxième (définition sociale) de l'École de Chicago.

13. Certes, le langage ordinaire introduit déjà une première forme de construction sociale. Ainsi, les expressions «conflit physique» et «agression physique» peuvent ne pas avoir la même connotation. Mais nous voulons distinguer ici ce niveau de construction sociale de la construction institutionnelle opéré par le droit / système pénal. 


\section{LES AUTRES OPPOSITIONS ET DIVERGENCES}

Ce débat entre les paradigmes a eu pour effet de nous faire oublier qu'il existe d'autres divergences en criminologie et qu'elles sont également importantes. Nous aimerions en souligner deux pour nos propos.

1) D'abord, on suppose souvent qu'il existe seulement deux conceptions de la personne humaine en criminologie: l'une qui voit l'individu comme un acteur faisant un choix libre et rationnel (pensée classique) et l'autre qui le voit comme un être entièrement déterminé (positivisme). Ces deux conceptions ont souvent un objectif commun: combattre certains types de situations-problèmes par la peine ou par des «mesures de sûreté». Le droit pénal (néo-)classique veut axer la défense sociale sur l'idée de libre arbitre, de responsabilité, de culpabilité, de peine, etc.; le droit pénal d'inspiration positiviste préfère parler plutôt de «mesures de défense sociale» que de peines et veut réadapter ou neutraliser plutôt que «punir».

Or, on n'est pas obligé de choisir l'une ou l'autre de ces vues. En effet, il existe une troisieme conception pour laquelle l'acteur n'est ni doué d'une rationalité et d'une liberté abstraites et universelles ni absolument déterminé par des traits, des pulsions, des tendances, etc. L'être humain, y compris l'infracteur, est ici un acteur situé: «dans la mesure où il est porteur d'un point de vue propre qui dépend de la position qu'il occupe dans le cadre social, de l'histoire qui a été sienne et des projets autour desquels son activité s'organise" (Debuyst, 1990, p. 26). Les personnes sont vues ici comme étant prises, si l'on peut ainsi dire, «dans des jeux de pouvoir» et, ayant à vivre «dans ses relations avec les autres à l'intérieur ou au-delà de ces jeux, des processus de reconnaissance ou de non-reconnaissance qui paraissent essentiels dans l'élaboration de sa propre identité" (Debuyst, ibid.). Les notions de rationalité, de responsabilité absolue et de déterminisme paraissent alors trop expéditives pour rendre compte convenablement de la complexité des situations. Cette troisième façon de voir est celle qui prend le plus de distances vis-à-vis de l'idéologie pénale passée et contemporaine. On la retrouve dans la psychologie et dans la sociologie phénoménologiques.

2) Le deuxième point est le suivant. On a maintes fois affirmé que la criminologie sociologique s'opposait à la criminologie clinique. Ce problème nous paraît aujourd'hui mal posé. En effet, la grande différence ne se trouve pas dans une opposition entre les points de vue des deux disciplines, mais plutôt dans les perspectives que chaque auteur 
adopte dans sa propre discipline. Bref, il ne s'agit guère d'un clivage transversal séparant la sociologie de la psychologie, mais bien d'un clivage horizontale entre les orientations dans chaque discipline. Il est plus facile à certains sociologues et psychologues de se comprendre entre eux qu'avec certains de leurs collègues.

Dans le champ pénal, cette prétendue opposition entre la sociologie et la psychologie (ou psychiatrie) a masqué particulièrement les divergences entre les différentes orientations dans la criminologie clinique elle-même. Le positivisme criminologique a non seulement donné une image faussement consensuelle du champ, mais il a surtout projeté une image consensuelle de la criminologie clinique en l'opposant en entier à la sociologie ou à une partie de celle-ci ${ }^{14}$. Or, dès les années vingt-cinq et trente, une orientation phénoménologique s'est développée en psychiatrie et en criminologie clinique (Debuyst, 1990, p. 27), qui met l'accent sur l'acteur situé et qui s'apparente davantage à la perception des sociologues de l'École de Chicago (1915-1960) plutôt que de la perspective clinique positiviste centrée sur la recherche d'une différence entre les délinquants et les non-délinquants. Cette orientation est mise au point au départ à l'École de Louvain avec Étienne De Greeff et a été adoptée après par l'École psychiatrique de Lyon, (Colin, Hochmann et Hijazi). Aujourd'hui, c'est surtout à l'École de Louvain que nous assistons au développement d'une criminologie clinique alternative qui prend très au sérieux la contribution faite par le paradigme de la définition sociale ${ }^{15}$. En plus, cette école participe activement au travail de construction d'un troisième paradigme visant à dépasser les deux précédents. Ceci montre que le paradigme de la définition n'est pas entièrement inutile, comme on l'a prétendu, d'un point de vue pratique et clinique. Inversement, on peut voir que la criminologie clinique contribue activement à la réflexion sociologique sur les paradigmes.

14. Debuyst (1986, p. 256) avait déjà souligné qu'il existe $\ll$ des criminologies cliniques et non pas une criminologie clinique ». Mais il était concerné par les effets de cette occultation envers les pénalistes.

15. Il y a bien sûr d'autre sites où une nouvelle perspective clinique se développe. On doit signaler particulièrement les travaux de Lode Walgrave (1986), en Belgique, et d'Uberto Gatti (1991), en Italie. Au Québec, on ne comptait jusqu'à présent qu'avec certains travaux de Laplante dirigés vers l'intervention $(1985 ; 1991 ; 1992)$. Paraîtra bientôt un travail de Serge Brochu sur Les drogues et la question pénale (titre provisoire) qui poursuit aussi cette orientation générale. 


\section{LA PREMIÈRE PHASE DE LA CRIMINOLOGIE CLINIQUE DE L'ÉCOLE DE LOUVAIN}

Pour bien apprécier la contribution de la criminologie clinique de l'École de Louvain à la construction d'un nouveau paradigme, il faut rappeler les positions qu'elle a prises lors de sa première période qui est demeurée, hélas, largement méconnue au Québec. Nous retiendrons quelques aspects de la pensée de De Greeff (1898-1961) pour faire ressortir les éléments de son approche qui illustrent nos propos.

\subsection{L'approche et l'option épistémologique de De Greeff}

De Greeff a fait des études en médecine et une spécialisation en psychiatrie. En 1926, il est nommé médecin anthropologue de la prison centrale de Louvain et, trois ans plus tard, il enseigne à l'École des Sciences criminelles de l'Université de Louvain qui vient d'ouvrir ses portes (Debuyst, 1991, p. 1).

1) La pensée de De Greeff est fondamentalement phénoménologique: il est particulièrement préoccupé par la manière de voir et le vécu, le « je», du sujet (Debuyst, ibid.). Le passage à l'acte est conçu, en règle générále, comme le résultat d'un processus normal et complexe d'adaptation de l'individu à une situation de vie. Dès lors, «le délit ne présente pas en soi un produit pathologique» (ibid., p. 2-3). Il exprime la relation de l'individu avec sa biographie et son milieu et se présente souvent, pour l'individu, comme la meilleure forme d'adaptation qu'il est susceptible d'envisager (De Greeff, 1956, p. 14; 1935). De Greeff montre que l'on peut saisir le processus de passage à l'acte sans faire appel à une «criminologie des facteurs». Son étude sur La psychologie de l'assassinat dans le cadre des "cas normaux» (De Greeff, 1935, p. 153) en constitue la preuve. Ici, comme le remarque Debuyst (1991, p. 3), «la question consiste à savoir comment un individu normal peut en arriver à supprimer une personne qu'il vit comme obstacle?». La base de son approche et de son intervention clinique se situe dans la recherche du sens du comportement pour l'individu. Nous dirions aujourd'hui que De Greeff est beaucoup plus intéressé par l'étude d'une relation sociale, dans ses aspects psychologiques, que par la recherche d'une invariante (traits de personnalité, engagement dans le crime, etc.) chez l'individu susceptible d'expliquer son être et ses comportements. Il n'est pas non plus intéressé par l'étude des opportunités du passage à l'acte en dehors de ce cadre relationnel. 
Sur le plan épistémologique général, la question clé est donc la suivante: "Comment arriver à connaître l'autre sans le réduire à l'état d'objet?» (Digneffe, 1989, p. 182) Lorsque cela s'applique à l'infracteur, la question devient: «Cet homme criminel, comment faut-il le voir et quelle attitude s'impose envers lui ?» (De Greeff, 1950, p. 269). Il ne faut pas dissocier cette question de la manière par laquelle De Greeff y répond. Pour lui, l'être humain n'est pas un objet et cette attitude se distingue aussi bien de celle de la criminologie positiviste que de celle de la pensée (néo-)classique utilitariste axée sur la dissuasion par la peine ${ }^{16}$.

2) D'une manière générale, on peut dire que De Greeff ne se sent à l'aise ni avec la vision déterministe adoptée par le positivisme ni avec celle du droit pénal classique sur le libre arbitre. «Si le déterminisme pratiqué dans les sciences biologiques n'est pas applicable sous la même forme à la psychologie, le concept de responsabilité, tel qu'il est défendu et brandi par certains représentants du droit, n'est pas davantage soutenable.» (De Greeff, 1931, p. 451) Il considère même que l'attitude de ceux qui «défendent à travers tout le principe de la responsabilité... est encore plus anti-scientifique que celle de ceux qui veulent nier ce fait » (p. 452). Car au moins les positivistes ont été poussés à s'y arrêter un instant pour réfléchir. Ce double refus l'amène à la conception de l'acteur situé.

3) L'approche de De Greeff se caractérise aussi par un esprit d'ouverture et d'autocritique par rapport à ses propres positions: «Tout au long de sa carrière de clinicien et de chercheur, De Greeff réfléchit sur le sens de sa propre démarche." (Digneffe, 1989a, p. 182) Cela ressort sans peine ${ }^{17}$ de son rapport au congrès de 1950 :

J'eus la chance inouie d'être médecin anthropologue dans une prison abritant 500 grands criminels... J'ai dû les suivre pendant dix et vingt ans et rectifier mes innombrables erreurs. Mes observations avaient été faites

16. Il y a une variante de la pensée pénale classique qui paraît, à première vue, converger avec la vision de De Greeff, mais qui est au fond aussi radicalement différente. C'est le rétributiviste de Kant et Hegel. La ressemblance tient au fait qu'ils s'opposent à l'utilitarisme et affirment que l'être humain ne doit pas être vi comme un moyen pour réaliser un autre bien (comme la dissuasion, par exemple). Hélas, ils ont une conception tellement naìve et abstraite du libre arbitre et de la responsabilité qu'ils succombent vite à l'idéologie pénale sur les peines fortes (Pires, 1990; 1991). Le conflit n'est pas conçu comme une relation.

17. Et pourtant l'un de nous, au cours d'une étude précédente (Pires, 1979), en voulant illustrer l'attitude générale de la criminologie à l'égard de la notion de crime, ne s'est pas rendu compte de l'originalité sous-jacente aux positions de De Greeff. 
avec suffisamment de soins pour que je me sente engagé par mes jugements, et maintenant je m'aperçois que la partie la plus intéressante de mon travail a résidé dans le besoin de m'expliquer ces erreurs, erreurs d'interprétation, erreurs de pronostic, erreurs dans l'affirmation de la guérison ou dans l'affirmation de la persistance du danger; innombrables erreurs... (De Greeff, 1950, p. 268-269)

4) Son épistémologie se caractérise ensuite par un double axiome: a) l'axiome de l'identité fondamentale des êtres humains, et $b$ ) celui de l'altérité phénoménale des expériences de vie (Debuyst, 1973, p. 284285; Digneffe, 1989a, p. 182). Le premier signifie que nous pouvons tous nous identifier d'une certaine manière à autrui du fait que nous partageons le même "drame humain " et une même "communauté de destin». La reconnaissance de cette identité fondamentale doit faire partie de la relation clinique et donne «au lien clinique une qualité faite de sympathie (sun-pathein) et de participation" (Debuyst, 1973, p. 284). Le deuxième axiome prépare le chercheur et le clinicien à une posture d'exploration qui est analogue à celle de l'anthropologue, en ce sens qu'il anticipe et suppose l'existence d'une différence dans la situation qu'il a devant lui. Pour De Greeff, l'autrui est alors différent de moi, «en ce sens qu'il voit et éprouve le monde "autrement" que moi" (Digneffe, 1989, p. 182). Bref, son épistémologie est marquée par un va-et-vient ou par le jeu conceptuel de l'identité et l'altérité. Autant du point de vue théorique que pratique, ce jeu conceptuel neutralise l' «étrangeté » attribuée à la déviance et anéantit la différence essentielle postulée entre les délinquants et les non-délinquants.

5) Ces axiomes sont accompagnés d'un objectif éthique et pratique: la réduction de la zone d'incommunicabilité entre les êtres humains (Debuyst, 1973). Il y a ici pour le clinicien, mais également pour le chercheur, une tâche capitale à accomplir: le dialogue en vue de comprendre les sujets et d'ouvrir les portes à une voie émancipatrice. Par définition, le clinicien veut permettre à son patient de "sortir» d'une situation de blocage déterminée dans laquelle il se trouve. Mais le paradoxe est que l'on ne doit pas cibler ni s'adresser exclusivement au patient, mais aux relations pertinentes: individu-proches, individugroupe social, etc. Car l'émancipation ne peut pas se faire sous le signe de la pure subordination de l'un à l'autre. Ceci réclame un regard critique bidirectionnel: à l'égard de l'individu et de la réaction sociale. Ce souci éthique, théorique et pratique pour le dépassement de l'incommunicabilité est présent chez De Greeff, mais est développé et approfondi davantage plus tard par Debuyst $(1969 ; 1989 a)$. 
6) Enfin, à tout cela s'ajoute une «vigilance épistémologique» (Bachelard) accrue à l'égard de tous les points de vue, de toute interprétation: vigilance à l'égard du regard du sujet sur le monde, mais également - et à plus forte raison — vigilance à l'égard du regard du monde sur le sujet. S'il faut se méfier des déformations, il faut aussi savoir qu'elles font partie intégrante de tout processus de (mé-)connaissance, de (non)communication, d'émancipation ou d'oppression et des jeux de pouvoir.

\subsection{De Greeff et la psychogenèse de la réaction de défense}

Par contraste avec nombre d'autres auteurs de son temps, la pensée de De Greeff n'a pas la connotation belliciste, stigmatisante et réductrice à l'égard du sujet qui caractérise les discours sur le crime. Au contraire, il a un regard bidirectionnel: il observe à la fois d'un œil empathique et critique les conséquences du comportement du sujet et ses justifications d'une part et la manière par laquelle la société «fait justice » et ses justifications d'autre part. Pour lui, aucun des deux côtés n'a entièrement raison, si l'on peut ainsi dire. Il choisit de bâtir les conditions pour un dialogue sur les conflits vécus plutôt que de se contenter d'un monologue sur la délinquance.

De Greeff est donc insatisfait de la justice pénale. Mais il ne faut pas se méprendre: son insatisfaction ne se rapproche ni de celle de Beccaria, ni de celle de Tocqueville, ni encore de celle de Garofalo. Il n'est pas insatisfait parce que cette justice ne sait pas bien punir, n'est pas assez punitive ou, au contraire, n'est pas assez correctrice. Il est insatisfait parce que cette justice est réductrice de l'être humain, stigmatisante, et parce qu'elle s'organise plus en fonction des instincts de défense et du sentiment d'injustice subie que des instincts de sympathie et de la valeur de justice. Si Mead (1917) a fait une contribution importante à la «psychologie de la justice punitive», De Greeff (1947) a emboîté le pas avec une psychogenèse de la réaction de défense.

En effet, comme le souligne Debuyst (1991, p. 4), l'êtude de De Greeff sur Les instincts de défense et de sympathie ${ }^{18}$ (1947), entre autres, a un intérêt pour le criminologue et le juriste car, à côté du comportement délinquant, De Greeff étudie aussi la réaction sociale et pénale. Que nous apprend-il sur cette question?

18. De Greeff est influencé dans le traitement de cette question par l'essai phénoménologique de Max Scheler, Nature et formes de la Sympathie, qui a été traduit de l'allemand chez Payot. 
1) Disons d'abord que De Greeff identifie deux grands groupes d' «instincts »: ceux axés sur la défense et ceux axés sur la sympathie. Il s'agit, en réalité, de deux types d'impulsions ou de réactions ${ }^{19}$. Ces réactions constituent à la fois deux modes de rattachement fondamentaux au monde et deux modes de connaissance d'autrui (Digneffe, 1989 , p. 187 ; Debuyst, 1991 , p. 7). La réaction de défense - qui a tendance à attribuer aux activités externes «dirigées» contre le sujet une intention hostile — produit une image ou une connaissance réductrice d'autrui. En revanche, la réaction de sympathie - caractérisée par l'amour parental ou sexuel - produit une image ou une connaissance valorisante ou sacralisante d'autrui :

Pour l'instinct de défense la forme «intention agressive» réduit infiniment la personnalité ainsi jugée, tandis que pour l'amour parental [instinct de sympathie] la valorisation exagère jusqu'à l'infini la valeur de l'objet. L'un est destructeur, l'autre est créateur. (De Greeff, 1947, p. 122)

Rappelons que De Greeff n'a pas une conception déterministe de ces réactions. Comme il s'agit d'une réaction émotionnelle, elle «permet une prise de conscience à l'occasion de laquelle le sujet peut réagir en suivant le sens de la réaction instinctive, ou au contraire, en cherchant à la bloquer» (Debuyst, 1991, p. 5). Cependant, par la force des choses, il serait amené à donner priorité aux réactions de défense. Pour échapper à ses effets réducteurs et destructeurs, le sujet doit faire un bon usage des mécanismes valorisants fondés sur les instincts de sympathie (De Greeff, 1947, p. 16). Ces deux manières de connaître ont donc quelque chose en commun: «Tous deux substituent à la personnalitéobjet une représentation qui semble en être l'image exacte et qui n'est que la projection d'un état subjectif.» (ibid.) On peut donc voir le monde et autrui à travers le prisme de la défense (image réductrice) ou à travers le prisme de la sympathie (image valorisante ou sacralisante).

2) Ces considérations forment la toile de fond à partir de laquelle De Greeff fait une critique de la réaction sociale à travers l'examen des notions de responsabilité et de justice. En effet, pour lui, la notion de responsabilité recouvre «deux réalités au départ fondamentalement

19. Qu'il suffise de dire à propos de la notion d'instinct, qu'elle était déjà tombée en déconsiđération à son époque et qu'il en était pleinement conscient. L'usage qu'il en fait n'a nien à avoir avec celui, plutôt abusif, où l'instinct constitue une sorte de * deus ex machina» (De Greeff, 1947, p. 2). L'instinct est ici alors « une sensibilité prététablie (c.-à-d. innê) à être émotionnellement sensible à certains stimuli» (Debuyst, 1991, p. 4). 
différentes: la responsabilité vécue et la responsabilité attribuée par le groupe social" (Debuyst, 1991, p. 5). La dernière est axée sur la réaction de défense et est souvent réductrice du sujet perçu comme agresseur. La première est plus complexe car, dans certains cas, le sujet, pour une série de raisons, a tendance à exagérer sa part de responsabilité et, dans d'autres, à la nier totalement. Ce n'est pas parce que le sujet se sent responsable qu'il l'est réellement ou entièrement; il ne suffit donc pas de lui faire avouer sa responsabilité pour l'établir convenablement ${ }^{20}$. Le clinicien ne peut alors se satisfaire entièrement ni avec ce que le sujet dit sur lui ni avec ce que les gens en réaction de défense disent sur le sujet. «Le vrai problème de la justice, remarque Debuyst (1991), est de faire en sorte que ces «deux réalités» se rejoignent.» Bref, la responsabilité «objective» reste donc quelque part entre les deux formes d'attribution, et on ne peut $y$ arriver que de manière approximative et toujours incertaine.

De Greeff $(1947$, p. 91) remarque que nous n'obéissons pas à une démarche de haute intellectualité lorsque nous parlons de responsabilité par rapport à nous-mêmes ou aux autres. Mais la psychogenèse de l'attribution de la responsabilité à une autre personne relève d'un autre ordre, dans la mesure où elle traduit une manière de se défendre (Debuyst, 1991, p. 6) et est souvent «la forme sociale que prend la connaissance de l'homme par l'instinct de défense " (De Greeff, 1947, p. 95).

Cette réaction de défense ne se manifeste pas seulement par le désir de se venger et de demander réparation pour l'injustice subie. Elle se traduira également par l'entrée en jeu de mécanismes psychologiques qui se traduisent par la projection sur l'auteur de l'atteinte subie d'une intention méchante et par la réduction de celui-ci à ne plus être qu'une responsabilité coupable... Dès lors, à côté de l'impulsion qui vise à réagir et à se venger, l'instinct de défense constitue en même temps - et ceci pour De Greeff est essentiel - un mode de connaissance, c.-à-d. une manière quasi-réflexe de projeter sur l'autre une image dans laquelle celui-ci n'est plus vu qu'à travers ses aspects négatifs et se trouve identifié à une volonté responsable qui justifie son élimination ou suppression. (Debuyst, 1991, p. 6)

Cette responsabilisation outrancière de l'autre se trouve autant dans le discours de l'infracteur que dans celui des tribunaux (ibid.). La position de De Greeff ici est loin d'être unilatérale. Il distingue alors la

20. Il ne faut pas confondre ici la responsabilité au sens plein du terme avec le simple constat factuel d'avoir ou non posé un geste (avoir tué, etc.). 
«valeur de justice» du «sentiment de justice». La première est une vertu difficile, le deuxième une impulsion qui nous induit en erreur si l'on ne fait pas un effort conscient pour surmonter le déterminisme instinctif de défense. Or, les gens ne doutent pas suffisamment de leur aptitude à distinguer le juste de l'injuste et ont souvent la certitude d'agir en toute justice (De Greeff, 1947, p. 35). Même ceux qui ont l'habitude de la réflexion, dit-il, n'y échappent que très imparfaitement (p. 36). On succombe vite au pur sentiment de justice lorsqu'il s'agit de juger la conduite d'un adversaire. Pour cheminer vers la valeur de justice, il faut, comme l'a joliment exprimé Debuyst (1991, p. 7), « désubjectiver le phénomène » et tenir compte de tous les points de vue.

Cependant, lorsque le groupe social réagit à une situation vécue comme une injustice subie, il ne lui est pas facile de tenir compte du point de vue du coupable «car il a l'impression de perdre ses moyens de defense et de renoncer à quelque chose qui lui paraissait «juste» (Debuyst, 1991, p. 7). Et le plus grave est que le sentiment de culpabilité ne semble pas exister par rapport à la notion de justice: l'individu moyen ne voit pas d'écart entre son aptitude actuelle à distinguer le juste et l'injuste et ce qu'il voudrait atteindre; «il ne se sent jamais coupable à ce point de vue et ne s'ameliore donc pas» (De Greeff, 1947, p. 39).

De Greeff remarque alors que la justice pénale et la connaissance qu'elle produit prennent souvent la forme sociale de cette réaction instinctive, outrancière et naïve :

Les parents normaux se gardent de tels excès, car l'amour parental inhibe l'agression et ne permet pas la réduction totale de l'enfant à l'état d'être responsable mais les notions de responsabilité qui leur servent dans la vie sociale ne sont pas plus compliquées que celles qu'ils utilisent dans leur vie familiale. Qu'ils soient médecins, magistrats, philosophes ou gendarmes, la responsabilité d'un homme augmente à leurs yeux en même temps qu'augmente la gravité ou la quantité de son activité anti-sociale [sic], c'est-à-dire exactement en même temps qu'augmente l'agressivité ou la peur à son égard. (De Greeff, 1947, p. 93-94).

Bien sûr, le «délinquant» est de tous les êtres celui sur lequel on a le plus tendance à porter un jugement simpliste :

Devant le tribunal il n'est rien qu'un faisceau d'intentions mauvaises et dans chacun de ses actes, même dans un acte socialement bon, on découvre sans peine l'intention antisociale, l'être ignoble qu'il faut punir. Rien n'est plus lamentable que cette reconstitution de l'homme sous le signe de la responsabilité pure. (ibid., p. 94-95) 
Ces remarques sont suffisantes pour montrer comment la vision de De Greeff ne reconduit ni les partis pris de la criminologie positiviste ni ceux de la criminologie classique. On peut dire que De Greeff a valorisé beaucoup l'idée de relation d'aide dans les rapports avec ses patients, mais beaucoup moins celle de réhabilitation qui a une connotation positiviste et suppose une pathologie. Il convient de distinguer ces notions pour construire une nouvelle manière d'envisager la pratique. Pour lui, d'ailleurs, le sujet est et demeure un sujet des droits. C'est dans ce sens qu'il faut comprendre son affirmation selon laquelle « dès qu'un individu a commis un délit, il appartient à la société avant d'appartenir à la médecine et il importe que l'affaire soit instruite et jugée» (De Greeff, 1931, p. 458).

\subsection{Louvain et Chicago : une brève comparaison}

La pensée de De Greeff a une grande affinité avec l'orientation sociologique de l'École de Chicago (1915-1960) qui se développe outre-mer. Cependant, il ne semble pas en avoir pris connaissance (Debuyst, 1991, p. 4). En effet, les deux écoles, Louvain et Chicago, adoptent une perspective phénoménologique, conçoivent la transgression comme étant essentiellement un processus d'adaptation normale des sujets à leurs conditions de vie, et accordent une place centrale à la relation empathique avec les sujets et à la notion charnière de "processus». D'un point de vue méthodologique, le «comment devient-on déviant? ? remplace le «pourquoi le crime? ? des classiques et le «qui est le délinquant?" des positivistes ${ }^{21}$. A fortiori, les deux écoles s'écartent du positivisme criminologique qui veut distinguer les délinquants des honnêtes citoyens. De Greeff (1935) étudie alors l'assassinat comme un processus psychologique de «conversion», tandis que Sutherland (1937), à peu près à la même époque, étudie Le voleur professionnel comme un processus sociologique d'engagement dans un métier. Les deux écoles et les deux disciplines montrent que dans tous les cas nous pouvons - et que dans la majorité des cas nous devons - nous référer aux activités des sujets, même dans le cas d'actes sérieux, dans le cadre d'un processus normal et en tenant compte de leur manière de voir ${ }^{22}$.

21. Certes, l'École psychiatrique de Lyon a aussi fait des réserves majeures a l'explication positiviste à partir de facteurs ou de traits (Hijazi, 1966, p. 83-106). Ce genre d'explication est vue comme ambiguë, très vague, de nature exploratoire, voire déroutante lorsque prise sans référence «à un cadre social bien déterminé» (Hijazi, 1966, p. 85).

22. Voir le parallele que nous faisons ici avec les remarques d'Alfred Schutz (1987, p. 43). 
L'axiome de l'identité fondamentale de De Greeff n'est pas sans trouver une certaine correspondance avec celui de l'appréciation empathique de l'École de Chicago et l'axiome de l'altérité, avec celui de la diversité. En effet, les "Chicagoans ${ }^{23}$ " anticipent aussi une certaine altérité dans le monde social du déviant et entendent la respecter et être en mesure de la refleter dans leurs analyses (Matza, 1969). Les deux écoles conçoivent aussi l'être humain comme un acteur situé, ne s'appuient pas sur une éthique belliciste et ne voient pas la peine ou les mesures de sûreté comme une solution aux problèmes sociaux ou à ceux des individus. L'analyse de De Greeff sur l'instinct de défense rappelle les réflexions de Mead sur la justice punitive de même que celles de Lemert (1951, p. 56) sur la « déviance putative» engendré par la réaction sociale ${ }^{24}$.

Signalons, en passant, que l'axiome de l'identité fondamentale trouve aussi une certaine affinité dans les épistémologies féministes. À cet égard, l' "épistémologie du point de vue des femmes» (Feminist Standpoint) a particulièrement souligné l'idée d'une expérience unitaire (voir Harding, 1986; 1987). En revanche, l'axiome de l'altérité a trouvé moins d'écho jusqu'à très récemment dans ces épistémologies, car l'accent sur l'expérience commune semblait l'emporter sur l'anticipation d'une expérience différente des sujets. En effet, les caractéristiques de race, de classe et de culture, bien que reconnues, étaient quelque peu théoriquement délaissées en faveur d'un accent mis sur la situation commune de domination dans une société patriarcale. Cependant, les études féministes axées sur une épistémologie postmoderne ont amené le feminist standpoint à anticiper théoriquement l'existence d'autres différences. On voit alors paraître des essais très importants qui tentent de faire le point entre la notion d'identité et celle d'altérité phénoménale réclamée par l'épistémologie féministe postmoderne à travers la notion de "fractured identities» (Cain, 1990). Il y a donc ici aussi un mouvement vers l'articulation d'un double axiome d'identification et de reconnaissance de l'altérité.

Bien sûr, une analyse plus fine de ces orientations reste à faire. L'important ici est cependant de montrer qu'avec les recherches féministes sur les comportements problématiques des femmes les

23. Les termes «Chicogoans» et «Neo-Chicagoans » ont êté employés dans un sens général pour désigner les sociologues qui ont adopté respectivement l'orientation de la première (1915-1960) et de la deuxième (après 1960) périodes / problématiques de l'École de Chicago. Voir Robert et Kellens (1973).

24. Pour Lemert, la déviance putative est celle qui est injustifiée parce qu'elle ne trouve pas une base dans le comportement objectif. Voir à ce sujet Rains (1975). 
orientations de l'École de Chicago et de l'École de Louvain forment une base commune à partir de laquelle une vision non positiviste et non classique de la criminologie peut se constituer.

\section{LA NOUVELLE CRIMINOLOGIE CLINIQUE DE L'ÉCOLE DE LOUVAIN (1973...)}

Christian Debuyst, qui étudie avec De Greeff, va poursuivre, actualiser et développer son orientation générale en tant que criminologue et psychologue clinicien. La présentation que nous avons faite de De Greeff fait déjà partie intégrante de la révision critique mise en ouvre par Debuyst. Sans doute les premiers travaux de Debuyst ${ }^{25}$ appartiennent aussi à la première période de cette école. Mais on aurait tort de voir une coupure radicale entre les deux périodes. Par ailleurs, les enjeux pour la criminologie, les questions qu' on se pose et les défis à relever se modifient rapidement après les années soixante et soixante-dix.

En effet, la deuxième période commence dans la première moitie des années soixante-dix lors de la réception de l'interactionnisme symbolique en Europe continentale. Debuyst $(1973 ; 1974)$ commence alors immédiatement à repenser l'objet de la criminologie clinique face à la mise en cause provoquée par le paradigme de la définition. Nous nous limiterons ici à quelques remarques genérales avant de souligner sa contribution sur le plan paradigmatique.

Comme nous l'avons vu, la pensée de De Greeff était déjà bien proche de celle de la première phase de l'École de Chicago. À l'instar de Mead, De Greeff avait même déjà en germe une réflexion avant la lettre sur la réaction sociale. Or, Debuyst se donne pour tâche de bâtir un pont entre la pensée phénoménologique de De Greeff sur la manière de faire déviante et la sociologie de la réaction sociale des «NeoChicagoans». Ce faisant, il a abouti aussi à bâtir un pont entre les deux périodes problématiques de l'École de Chicago. Car Debuyst veut tenir compte des deux dimensions de la question: la genèse des situationsproblèmes et la téaction sociale et pénale à leur égard. Par ailleurs, à la différence de l'École de Chicago, il met en valeur les aspects historiques et macro-sociologiques d'autres orientations sociologiques. En outre, en tant que clinicien, il est intéressé par l'émancipation des sujets. Mais il ne réduit pas le problème du crime au traitement des individus. Les conflits ont clairement une dimension sociale et, comme

25. Voir particulièrement Debuyst (1960). 
pour De Greeff, la relation d'aide - dans le cas où elle est pertinente ne se confond pas avec la connotation positiviste donnee à la notion de réadaptation. Le clinicien doit avoir même une vision nuancée et complète (dans ses dimensions sociales) de chaque situation spécifique (Debuyst, 1991, voir aussi Laplante, 1992). Le sujet est vu encore davantage comme un acteur situé, et il souligne la nécessité pour le chercheur et le clinicien de prendre de la distance vis-à-vis d'une certaine «vision pénale du monde ${ }^{26}$ ». Il entreprend d'ailleurs lui-même une critique de la notion d'infraction (Debuyst, 1985) en faisant ressortir les aspects du problème qui ont un intérêt pour le clinicien.

Debuyst (1991, p. 107) remarque que l'ancienne définition de la criminologie comme «l'étude du crime et du criminel » est trop restrictive. Elle s'accommode bien aux pensées classique et positiviste, mais elle exclut les recherches sur la création et l'application des lois qui font partie du paradigme de la définition sociale. Elle est aussi inappropriée en ce sens plus fondamental qu'elle est l'expression d'une vision des sujets et du droit-système pénal qui n'est plus convenable. La spécificité de la criminologie semble résider pour Debuyst dans les relations complexes entre certaines manières de faire et une manière politique de définir et d'intervenir. Il ne faut pas alors supposer que les manières de faire qualifiées de «crime» aient une spécificité ontologique propre. Et on doit tenir compte de tout cela dans le cadre de la relation clinique (Debuyst, 1989). Il existe sans doute "plusieurs criminologies" (Debuyst, 1986; 1922), puisqu'il existe différentes manières de voir le problème et le sujet dont elle s'occupe, mais ces clivages ne sont pas dus aux disciplines mais aux choix qui s'operent sur les plans conceptuel, politique et éthique.

\subsection{La recherche d'un nouveau paradigme}

À notre connaissance, c'est avec Debuyst à l'École de Louvain, pour la première fois, qu'on a voulu renouveler la criminologie clinique de manière à tenir compte de la contribution faite par le paradigme de la définition sociale. Mais ce projet propre au départ à la criminologie clinique a pris vite une autre dimension. Car Debuyst s'est rendu compte que pour repenser la place de la criminologie clinique - qui s'est développée comme toute la criminologie dans le cadre du paradigme du fait social - en tenant compte de l'apport de la sociolo-

26. Gatti (1991), un criminologue clinicien italien, insiste également beaucoup sur la nécessité de ce recul critique par rapport à l'idéologie pénale, autant sur le plan théorique que pratique. 
gie de la réaction sociale (paradigme de la définition), il fallait dépasser certaines limites des deux paradigmes.

En effet, la criminologie clinique ne pouvait pas abandonner complètement 1'étude de la manière de faire. En revanche, Debuyst a vu que la dimension relative à la manière de définir et de réagir était également capitale. Donc, pour être bien fait, ce projet pour repenser la criminologie clinique ne pouvait pas se contenter d' «additionner» un paradigme à l'autre. On en vint alors peu à peu à prendre conscience de la nécessité de bâtir un nouveau paradigme susceptible de prendre en compte les deux dimensions du champ pénal - la manière de faire et la manière de définir - et les différents sujets qui y participent: l'auteur de l'acte, la victime immédiate, les agences officielles, le « groupe social», etc.

Le paradigme de la définition sociale avait proposé l'étude de deux aspects du processus de criminalisation : la question de la création et du maintien des lois pénales et celle de l'application des lois et ses conséquences (Baratta, 1975, p. 59; Robert, 1981, p. 274-276). Debuyst retient ces deux aspects, mais propose de les articuler autour de la notion d' "acteur social » afin d'intégrer la dimension relative à l'étude des situations conflictuelles (manière de faire). Il identifie alors «deux niveaux ${ }^{27}$ » : celui de la transgression de la loi (du conflit) et celui de la loi elle-même (création et application). Bref, le niveau de la manière de faire et celui de la manière de définir et de réagir.

Que l'on ne se méprenne pas. L'intention n'est pas celle de faire un «collage» entre les paradigmes, mais l'operation de construction d'un nouveau paradigme n'est pas aisée et réclame plusieurs essais ${ }^{28}$. Debuyst est probablement le premier à avoir proposé la notion d'acteur social comme un concept théorique visant à construire un troisième paradigme susceptible d'articuler les deux dimensions de la «question criminelle " : les relations entre les individus dans la société civile et les relations entre le droit pénal (l'État) et les individus.

27. En réalité, Debuyst hésite quant au fait de parler de deux ou de trois niveaux. En effet, on peut parler du niveau de la création de la loi, de celui de son application, et du niveau de la transgression et des conflits (Debuyst, 1990, p. $27 ; 1991$, p. 108). Mais la différence la plus importante est entre les deux premiers niveaux et le troisième. C'est en partie pour souligner cela que nous parlons ci-dessous plutôt de deux axes.

28. Il faut parler ici de dépassement (Aufhebung) au sens donné par Hegel. Le dépassement a lieu lorsqu'à partir de deux thèses antagonistes on arrive à en dégager une troisième (synthèse) qui retient certains élements des thèses précédentes, mais à un niveau plus élevé de compréhension. La nouvelle synthèse ne s'identifie pas alors aux thèses précédentes et n'est pas le résultat d'une simple addition des thèses (contraires) de départ. 


\section{LES ÉTUDES FÉMINISTES SUR LES COMPORTEMENTS PROBLÉMATIQUES DES FEMMES}

Du côté de la sociologie, les contributions les plus stimulantes pour un travail de réflexion théorique sur les paradigmes viennent à notre avis des études féministes sur les comportements problématiques des femmes. Et pourtant, ces études sont les moins visibles de toutes les contributions féministes dans le champ pénal.

Parent (1991) a démontré qu'il n'y a pas une ligne de démarcation nette et significative entre les études sur les comportements problématiques des femmes (fait social) et celles sur la réaction sociale à leur égard (définition sociale). Certes, cette distinction peut parfois se faire, mais elle n'est pas tranchée et ne semble pas appropriée au projet théorique féministe dans ce champ. En effet, les études féministes éprouvent une certaine difficulté à s'ajuster à ce clivage entre les deux paradigmes, et pour cause. La distinction la plus marquée dans le champ pénal est celle qui sépare les études sur la «déviance» des femmes de celles sur les femmes victimes d'agression (Parent, 1991) ${ }^{29}$.

Sur la base de ces résultats, nous proposons alors de distinguer deux grands axes d'études féministes en matière pénale: $a$ ) celui portant sur les comportements problématiques des femmes et le rôle du droitsystème pénal à leur égard; et $b$ ) celui portant sur les femmes en tant que victimes et sur le rôle du droit-système penal à leur égard. À l'heure actuelle, cette distinction se justifie par le fait que ces axes ne se placent pas en général de la même manière vis-à-vis des theories de la peine, n'ont pas la même perception du fonctionnement du système pénal et ne demandent pas des réformes sur les peines dans la même direction politique. Bien sûr, il existe aussi des points de croisement. Les deux axes identifient des préjugés sexistes dans le droit-système pénal, et partagent un même projet politique pour l'émancipation de toutes les femmes et pour l'élimination des rapports de domination patriarcale.

Il faut aussi remarquer que le degré de communication entre ces deux axes n'est pas égal dans les deux directions. La distinction ici est plus subtile et difficile à démontrer. En effet, nous avons l'impression que les études sur la victimisation des femmes, menées en bonne partie par des juristes féministes peu familiarisées avec la documentation critique en criminologie ou peu critique à l'égard de l'idéologie pénale,

29. C'est d'ailleurs pour cette raison que Parent (1991) a pu exclure les premières études de sa propre recherche qui porte sur la contribution féministe aux enjeux paradigmatiques en criminologie. 
souvent ne pensent pas en même temps à ce qui arrive, ou peut arriver, aux femmes qui sont criminalisées lorsqu'elles proposent des réformes visant à augmenter les peines. En revanche, les études sur les comportements problématiques des femmes et sur leur criminalisation, menées souvent par des féministes au fait de la documentation critique dans le champ, voient plus clairement les limites du droit pénal et en même temps les problèmes soulevés par la nécessité de mieux protéger les droits des femmes en général. Les travaux de Marie-Andrée Bertrand sont exemplaires à cet égard. Dès la fin des années soixante-dix, elle a manifesté un intérêt pour un nouvel encadrement théorique susceptible de saisir les comportements problématiques des femmes tout en étant, en même temps, très critique à l'égard de la réaction pénale dans son ensemble (vis-à-vis de la femme et de l'homme justiciables). En outre, elle était bien consciente du problème de la violence faite aux femmes, mais aussi des dilemmes, des coûts et des insuffisances entourant l'intervention pénale.

Les études sur les comportements problématiques des femmes et le droit pénal forment donc (encore) un axe avec une configuration propre. Nous sommes présentement convaincus qu'il constitue un siège privilégié pour une reconstruction globale de la problématique dans le champ pénal. L'étude de Parent (1992) nous permet de prendre conscience d'un certain nombre d'analogies et de contributions originales de cet axe. Ces études demeurent d'abord fideles à une perspective féministe dans la mesure où elles examinent les stéréotypes sexistes et les rapports de domination à l'égard des femmes aux niveaux de la structure sociale, du savoir et de la justice pénale, et qu'elles se préoccupent de l'émancipation des femmes. Elles font aussi une critique du positivisme criminologique: les femmes justiciables ne sont représentées ni comme des ennemies de la société, ni comme des personnes malades, ni comme des êtres différents des autres femmes (Parent, 1992). Le terme «réadaptation " est aussi délaissé en faveur de l'idée qu'il faut plutôt leur «donner du pouvoir » pour qu'elles puissent se prendre en main.

La critique du droit pénal classique est peut-être moins explicite, mais est également incontournable. Nous n'avons pas vu ces études vouloir gérer les situations-problèmes des femmes par l'entremise de peines fortes: peine de mort, longues peines d'emprisonnement, etc. L'image qui ressort des études biographiques sur la femme criminalisée est celle d'un sujet situé: ni entièrement déterminée ni posant un choix libre et rationnel (et donc «entièrement responsable»). On arrive même à proposer une version féministe de l'abolitionnisme (Harris, 1987). 
Parent (1991) a démontré que la pratique de la recherche đonne de plus en plus un portrait qui ne cadre facilement avec aucun des deux paradigmes, et ce, malgré le fait qu'on ne fait pas encore, en règle générale, une critique soutenue de la notion de crime. C'est que ces recherches produisent une mise en cause de la «femme criminelle» d'une part et refusent de quitter le terrain de l'explication des situationsproblèmes d'autre part. Mais les conséquences de ce cheminement sur le plan des paradigmes ont été moins exploitées.

Parent montre aussi comment ces études, dans leur ensemble, contribuent à bâtir un pont entre les deux phases de l'École de Chicago tout en corrigeant leurs biais et leurs écueils. On voit donc les liens qui peuvent se tisser, et qui sont en train de se tisser, entre l'École de Chicago, l'École de Louvain et les études féministes sur les comportements problématiques des femmes sur le plan de la recherche.

Pour des raisons d'ordre divers, ces études féministes sont demeurées en partie occultées. Elles ne satisfont pas les exigences du paradigme de la définition parce qu'elles ne sont pas assez constructivistes; elles ne satisfont pas le positivisme criminologique parce qu'elles sont trop proches de Chicago et ne partagent pas la même conception du "délinquant » et du droit pénal; elles ne satisfont pas non plus le néoclassicisme parce qu'elles se refusent à culpabiliser et à responsabiliser les femmes de manière abstraite et n'adoptent pas les théories rétributivistes ou de la dissuasion des peines; elles sont embarrassantes pour une bonne partie des études sur la victimisation des femmes parce qu'elles approfondissent la critique du pénal et ne partagent pas la vision belliciste des théories de la peine, etc. Bref, ces études n'ont pas présenté d'attrait pour nombre de perspectives et d'auteurs, car elles ne veulent pas simplement «ajouter les droits des femmes au droit pénal existant », mais modifier profondément ce droit vers la modération dans le même mouvement qu'elles essaient de faire valoir les droits des femmes sur le plan du droit étatique en géneral et sur le plan d'une nouvelle éthique sociale. Le droit pénal est vu ici comme discriminatoire non seulement à l'égard des femmes, mais des groupes ethniques et des classes défavorisées. Il est aussi un «instrument-massue» (Dumont, 1986, p. 18) qui provoque souvent des contrecoups à l'égard des femmes justiciables, des femmes victimes des groupes ethnicisés, des classes défavorisées, etc.; bref, il fait partie du problème et non de la solution.

Le modele que nous proposons ci-dessous veut tenir compte, entre autres choses, de ces deux orientations occultées, parfois même 
oubliées: la criminologie clinique de l'École de Louvain et les études féministes sur les comportements problématiques et la criminalisation des femmes. Bien sûr, il veut aussi tenir compte de la contribution faite par les deux traditions de l'École de Chicago et par l'ensemble des recherches du paradigme de la définition sociale. Qui plus est, ce modèle permet d'intégrer la question des rapports de pouvoir entre les groupes et donc celle de la victimisation des femmes et des autres personnes.

\section{VERS UN PARADIGME DES INTERRELATIONS SOCIALES ?}

Un des problèmes théoriques majeurs de la criminologie depuis la publication du livre de Taylor, Young et Walton (1973) a été celui de résoudre le paradoxe posé par ces deux paradigmes. On a l'impression, à juste titre, que chacun d'eux mutile la réalité. Cependant, il n'était pas clair au départ si on devait «additionner» un paradigme à l'autre ou si, au contraire, on devait chercher une nouvelle manière de poser le problème. À l'époque, ces auteurs ont proposé qu'on étudie la déviance selon un modèle à deux dimensions, mais ont donné l'impression qu'il s'agissait d'ajouter l'une à l'autre et de constituer un «champ d'etude bicéphale» (Robert, 1981, p. 262). Le modele proposé avait sans doute l'avantage d'indiquer la double dimension du crime, mais le grand désavantage de suggérer un modele dichotomique où l'on reste sans savoir qui a priorité sur quoi et quelles sont les conséquences théoriques de l'intégration proposée. Aucune appellation particulière ne sera par ailleurs donnée à ce modèle. Par la suite, on compte une série de contributions importantes mais, en règle générale, l'articulation de ces deux dimensions n'a pas fait beaucoup de progrès. D'ailleurs, à notre connaissance, avec les exceptions notables de Baratta $(1975 ; 1983)$ et de Debuyst $(1973 ; 1985 ; 1990 \mathrm{a} ; 1990 \mathrm{~b})$, tous ceux qui ont essayé d'intégrer les deux modèles ont glissé du côté du paradigme du fait social. Au début, Baratta (1975, p. 44) avait parlé de « deux éléments de la question criminelle» pour souligner la nécessité de dépasser les deux paradigmes précédents et a attiré l'attentisme sur l'apport fondamental du paradigme de la définition.

Inspiré par Baratta, Pires a utilisé l'appellation «double question criminelle » pendant longtemps dans le cadre de son enseignement pour désigner le troisième paradigme. Certes, nous étions tous, Baratta y compris, insatisfaits par la connotation dichotomique qu'elle continuait à véhiculer. Au début des années quatre-vingt-dix, Debuyst ouvre une 
voie permettant de sortir de l'impasse: il propose d'appliquer dans le champ pénal la notion d' «acteur social " mise en valeur, entre autres, par Touraine (1984). Cette «gageure» (Debuyst, 1990, p. 21), on peut déjà le dire, a permis d'effectuer un premier déblocage important dans la réflexion épistémologique sur les paradigmes. Nous avons alors parlé, provisoirement, de paradigme des acteurs sociaux pour désigner cette troisième manière de concevoir l'objet de la criminologie.

Les avantages que présente cette notion, par rapport aux tentatives précédentes, ont été indiqués par Debuyst lui-même. C'est que cette notion permet d'intégrer toutes les dimensions et tous les niveaux d'analyse. On peut parler des acteurs sociaux qui crént la loi ou qui essaient de la conserver, des acteurs sociaux qui appliquent les lois et des acteurs sociaux qui les transgressent ou qui sont en conflit entre eux dans la société civile. En plus, elle évite l'image d'une simple addition entre deux paradigmes, à condition que l'on respecte le cadre théorique proposé par Debuyst.

Certes, la notion d'acteur social pose encore un certain nombre de difficultés sur lesquelles il va falloir revenir. Une des questions que l'on peut se poser alors est de savoir si l'on doit retenir cette notion pour désigner le troisième paradigme ou si l'on doit chercher une autre désignation moins spécifique et exploratoire ${ }^{30}$. Quoi qu'il en soit, il y a une autre appellation, qu'on retrouve aussi chez Debuyst (1990, p. 28), qui nous paraît plus appropriée pour désigner la troisieme conception de l'objet: la notion d'interrelations sociales. Nous avons alors, en gros, trois manières de concevoir l'objet de la criminologie (ou des sciences sociales en général):a) comme un fait social $; b$ ) comme une définition sociale; ou $c$ ) comme une relation sociale, comme des inter-relations sociales (Pires, 1992).

C'est au cours d'une discussion avec Fritz Sack, engagé à partir d'un texte de Pires (1992), que Sack a attire l'attention sur cette notion. Sack a reconnu la nécessité de traiter des deux dimensions comprises dans la notion de crime, mais a également beaucoup insisté sur la nécessité de les traiter sous le signe de «relations», de «rapports». Ceci aurait l'avantage de relativiser à la fois la notion de comportement et de définition. De là est venue alors l'idée de parler de paradigme des relations sociales. Par la suite, en relisant Debuyst, il est apparu que

30. La notion acteur social a aussi l'inconvénient d'être utilisêe par les perspectives néo-classiques, comme celle du choix rationnel. Mais cet inconvénient est incontoumable puisque aucune perspective n'a une chasse-gardée sur ses concepts. Il faut toujours les situer dans leurs cadres théoriques respectifs. 
l'idée de relations sociales était aussi au cour de son travail de reconstruction théorique, bien qu'en filigrane. Lorsqu'on «découvre » cette notion, on arrive difficilement à lire autrement ses travaux ${ }^{31}$.

Nous ne pouvons pas ici justifier épistémologiquement le modèle propose $e^{32}$, mais nous soulignons qu'il s'inspire aussi de manière importante de la sociologie de Bourdieu dans ses efforts pour transcender les frontières disciplinaires et les réductions mutilantes qui traversent les sciences sociales, aussi bien que pour construire un mode de connaissance susceptible de dépasser l' «antinomie entre physique sociale et phénoménologie sociale» (Wacquant, 1992, p. 16). Comme le remarque Wacquant (p. 23), la sociologie de Bourdieu «proclame le primat des relations " et celles-ci sont vues comme constituant la réalité sociale. Il s'agit donc en quelque sorte de développer cette perspective relationnelle par rapport au champ pénal. Signalons qu'il y a aussi déjà un bon nombre de recherches où le «crime » est représenté comme un problème de relations et de transformations sociales diverses. Certes, ces recherches n'ont pas exploré théoriquement cet aspect, mais elles se prêtent facilement à une relecture dans ces termes. On peut les voir comme des exemples de la réalisation de ce troisième paradigme. Nous les illustrons ici avec deux recherches.

La première est celle de Sack (1984) lui-même, qui montre comment le mouvement d'étudiants des années soixante-cinq en Allemagne a donné lieu à des événements de violence vécus et définis comme terroristes vers la fin des années soixante-dix. Ce processus d'«escalade» est étudié sous la forme d'un jeu d'interrelations sociales entre différents protagonistes et sous la forme de transformation de ces relations. L'analyse de cause à effet est exclue explicitement du cadre théorique et méthodologique (voir p. 87-92). En outre, le «terrorisme» n'est conçu ni à proprement parler comme un fait social consistant ni comme une simple définition sociale. Il est plutôt le résultat d'interrelations sociales, et Sack analyse les mécanismes, les intêrêts politiques, les stratégies, le processus de communication et les intentionnalités qui ont contribué à ce résultat sur le plan événementiel.

La deuxième est celle de Poupart (1979), qui étudie la genèse, le maintien et la régulation de la violence au jeu de hockey comme une

31. Cette notion d'interrelations sociales s'applique également bien à une relecture critique du droit criminel, mais cette question ne peut pas être traitée ici. Elle correspond à un nouveau paradigme éthique (Pires, 1991).

32. L'un de nous a déjà entamé cette réflexion ailleurs (Pires, 1990b). Un essai sur cette question doit paraître bientôt. 
forme d'interrelations sociales dans un contexte où la construction pénale est virtuellement gardée à distance, voire exclue. Sa recherche n'appartient ni au paradigme du fait (même s'il étudie la dimension phénoménale de la violence) ni à celui de la définition (même s'il étudie la manière de percevoir et de réagir vis-à-vis de ce phénomène) ${ }^{33}$. Cette violence, qui aurait pu devenir crime, est à la fois tout à fait publique et visible - on peut la voir à la télévision - et réglée par une autre pratique d'objectivation du phénomène. Et Poupart nous montre comment elle est produite (genèse) et gérée (réaction) par un autre système de règles dans le cadre de l'organisation interne du jeu de hockey.

Le modèle que nous proposons ci-dessous peut rendre facilement compte de ces pratiques de recherche. En outre, la notion de relations est aussi au cœur de la contribution féministe: les relations sociales et de pouvoir dissymétriques entre les femmes et les hommes. Le comportement problématique des femmes ou la violence à leur egard sont aussi des questions qui se laissent appréhender par ce cadre théorique, aussi bien que le traitement des justiciables (d'un sexe ou de l'autre) par le droit et le système pénal. Les analyses féministes ont aussi mis à l'ordre du jour la question des deux sortes de relations de pouvoir: celles qui se tissent entre les individus et groupes d'individus dans la société civile et celles, non moins importante, qui se nouent entre l'État et les (groupes d') individus. Qui plus est, certaines études théoriques féministes ont récemment mis en valeur explicitement cette notion sur le plan épistémologique (Cain, 1990).

En posant le problème en termes de relations et d'interactions, le comportement n'est pas exclu, mais devient, pour ainsi dire, une particule élémentaire du problème. Le véritable objet est l'étude des interrelations sociales et de la transformation de ces relations. Il convient de donner au terme «transformation» un double sens: il y a transformations dans les perceptions et représentations au cours des interactions (conflictuelles), et transformations dans la dimension phénoménale des relations elles-mêmes (pour le meilleur ou pour le pire). Nous ne pouvons pas développer davantage cette question ici. Qu'il suffise pour l'instant de dégager un modèle schématique de ce nouveau paradigme aux fins d'exploration.

33. Pendant son enseignement, Pires a demandé à ses étudiants à maintes reprises de situer cette étude de Poupart dans le débat paradigmatique. Trois positions émergeaient alors régulièrement: celle qui la voyait comme «étiologique», celle qui la classait comme une étude de la réaction sociale et celle qui hésitait entre les deux. Les trois positions ont leur raison d'être, car cette étude dépassait les modèles thériques existants. 
Le schéma ci-dessous résume les grandes lignes de ce modele. Les réflexions de Debuyst et une série de termes proposés par lui (acteur social, interrelations, etc.) nous ont servi de toile de fond. Dans sa forme simplifiée, ce modèle a deux grands axes. L'axe vertical comprend les rapports complexes et interactifs entre l'État et la société civile. C'est par l'entremise de cet axe que le droit-système pénal «construit» le crime par l'objectivation d'une pratique institutionnelle. On y trouve la problématique de la création de la loi (criminalisation primaire) et de son application (criminalisation secondaire), et celle des rapports de pouvoir entre l'État et les individus. La flèche verticale est bidirectionnelle puisqu'elle se réfère aussi bien à l'action de l'État sur la société civile à travers la création et l'application de la loi (personnel de l'État), qu'à l'action de la société civile sur l'État par l'entremise des groupes de pression. L'axe horizontal, en revanche, comprend les conflits entre les individus et les groupes d'individus: rapports sociaux entre les sexes, entre le «consommateur» et les «entreprises commerciales », entre la victime et l'infracteur, etc. On y trouve la problématique des situations-problèmes, de la transgression, et celle des rapports de pouvoir entre les individus et groupes d'individus. Bien sûr, le jeu de relations est complexe et on joue souvent sur plus d'un plan à la fois. Notons que l'on peut ajouter un autre axe horizontal, ou modifier les termes de cet axe, pour désigner, si cela est l'objet de notre recherche, les rapports entre sociétés ou entre "nations». Cette adaptation est nécessaire pour traiter, par exemple, la question des Inuit et des relations qui s'établissent entre les cultures (relations de dépendance et de colonisation). On peut alors rendre compte aussi de certaines pratiques nouvelles de recherche, comme celle de Jaccoud (1992).

Dans ce paradigme, le «crime» émerge comme le résultat de pratiques interactives et d'objectivation d'ordre diverse: manière d'agir et de définir entre les individus, et manière d'agir et de définir sur le plan institutionnel de l'organisation des droits. La dichotomie «comportement sans définition " et «définition sans comportement » est au moins partiellement dépassée au profit de deux dimensions relationnelles: d'une part, les relations et transformations de ces relations entre les personnes et, d'autre part, les relations spécifiques entre les personnes et l'État (le droit pénal). On peut voir cette réflexion comme l'aboutissement d'un travail collectif et comme un effort inachevé pour éclairer les nouvelles pratiques de recherche. Il faut maintenant développer, corriger, élargir, tirer les différentes conséquences et puis dépasser ce modèle lui-même. Mais ceci est une autre histoire. 


\section{BIBLIOGRAPHIE}

ACOSTA, F. (1988), «À propos des illégalismes privilégiés. Réflexions conceptuelles et mise en contexte», Criminologie, vol. $21, \mathrm{n}^{\circ} 1$, p. 7-34.

BARATTA, A. (1975), «Criminologia liberale e jdeologia della difesa sociale, $\mathrm{La}$ questione Criminale, 1, (1), p. 7-65.

BARATTA, A. (1983), Sur la criminologie critique et sa fonction dans la politique criminelle, rapport présenté au $\mathrm{IX}^{\mathrm{e}}$ Congrès international de criminologie (Vienne).

BERGER, P. et LUCKMANN, Th. (1966), La construction sociale de la réalité, Paris, Méridiens Klincksieck, 1986.

BERTRAND, M.-A. (1979), La femme et le crime, Montréal, L'aurore.

CAIN, M. (1990), « Realist phitosophy and standpoint epistemologies or feminist criminology as a successor science $"$, in L. Gelsthorpe et A. Morris (sous la direction de), Feminist perspectives in criminology, (p. 124-140), Milton Keynes, Open University Press.

COLLECTIF (1990), Acteur social et délinquance. Hommage à Christian Debuyst, Bruxelles, Mardaga éditeur.

COULON. A. (1987), L'ethnométhodologie, Paris, PUF.

DEBUYST, C. (1992), «Théories et pratiques criminologique», Criminologie, vol. 25, $\mathrm{n}^{\circ} 2$, (dans ce numéro).

DEBUYST, C. (1991), Étienne de Greef (1898-1961), Dictionnaire des criminologues belges (titre provisoire; en preparation), École de criminologie flamande de Louvain.

DEBUYST, C. (1990a), «Présentation et justification du thème», in Acteur social et délinquance. Hommage à Christian Debuyst, (p. 21-33), Bruxelles, Mardaga éditeur.

DEBUYST, C. (1990b), «Acteur social et délinquance », Diogène, vol. 150, p. 97-120.

DEBUYST, C. (1989), «Perspectives cliniques en criminologie. Le choix d'une orientation», Revue internationale de criminologie et de police technique, vol. 4, p. $405-418$.

DEBUYST, C. (1986), «Le droit pénal et les différentes problématiques possibles en criminologie ", Revue internationale de criminologie et de police technique, vol. 2, p. 256-275.

DEBUYST, C. (1985), Modèle éthologique et criminologie, Bruxelles, Mardaga éditeur.

DEBUYST, C. (1975), «Les nouveaux courants dans ta criminologie contemporaine. La mise en cause de ta psychologie criminelle et de son objet , Revue de droit pénal et de criminologie, vol. 10, p. 845-870. 
DEBUYST, C. (1973), «Une criminologie de l'étiquetage ou d'une criminologie du passage a l'acte? Un problème que nous pose l'attitude clinique du $\mathrm{D}^{r} \mathrm{E}$. De Greff $\%$, Annales internationales de criminologie, vol. 12, ( $\mathrm{n}^{\circ} 1$ et 2), p. 283-290.

DEBUYST, C. (1969), «La mise en cause des règles sociales dans la littérature», Annales de droit (littérature et criminologie), vol. 29, $\mathrm{n}^{\circ} 2$, p. 113-140.

DEBUYST, C. (1960), Criminels et valeurs vécues, Louvain et Paris, Nouwelaerts, $2^{e}$ éd.

DE GREEFF, E. (1956), « Sur le sentiment de responsabilitê », Revue internationale de défense sociale, vol. 10, p. 1-19.

DE GREEFF, E. (1950), "Criminogenèse, rapport Actes du IF congrès international de criminologie, (p. 267-306), Paris, PUF, 1955.

DE GREEFF, E. (1947), Les instincts de défense et de sympathie, Paris, PUF.

DE GREEFF, E. (1935), « La psychologie de l'assassinat * (1 $1^{\text {tre }}$ partie), Revue de droit pénal et de criminologie, $15,(2)$, p. 153-164; (2e partie), vol. $15, \mathrm{n}^{\circ} 3$, p. 213 235 ; ( $3^{*}$ partie), $15,(4)$, p. $357-396$.

DE GREEFF, E. (1931), * La notion de responsabilité en anthropologie criminelle», mémoire, Revue de droit pénal es de criminologie, vol. 11, p. 445-460.

DELRUELLE-VOSSWINKEL, N. (1981), "La recherche-action: un nouveau paradigme de la sociologie ? », Revue de l'Institut de sociologie, vol. 3, p. 513-527.

DIGNEFFE, F. (1989a), «La perspective de E. De Greeff», Déviance et Société, vol. XIII, nª 3, p. 181-198.

DIGNEFFE, F. (1989b), Ethique et délinquance. La délinquance comme gestion de sa vie, Genève, Paris, Médecine et Hygiène, Méridiens.

DUMONT, H. (1986), Le contrôle judiciaire de la criminalité familiale, Montréal, Thémis.

GATTI, U. (1991), «L'intervention psychosociale et la justice des mineurs: modèles d'intégration ou de séparation $m$, in L'intervention psychosociale au sein du pénal, Actes de la $1^{\text {ère }}$ Conférence internationale du GREPO, vol. 1, département de criminologie, Université d'Ottawa.

HAAN, W. de (1990), The Politics of Redress. Crime, Punishment and Penal Abolition, London, Unwin Hyman.

HAAN, W. de (1992), «Redresser la situation-problème. L'abolitionnisme et le controle de la criminalitê, Criminologie, vol. $25, n^{\circ} 2$, (dans ce numéro).

HARDING, S. (1987), *Conclusion. Epistemological Questions", in S. Harding (sous la direction de), Feminism and Methodology (p. 181-190), Milton Keynes, Open University Press.

HARDINGS, S. (1986), The Science Question in Feminism, Milton Keynes, Open University Press.

HARRIS, M.K. (1987), «Moving Into the New Millenium: Toward a Feminist Vision of Justice», The Prison Journal, vol. 67, p. 27-38. 
HIJAZI, M. (1966), Délinquance juvénile et réalisation de soi, Paris, Masson Éditeurs.

HULSMAN, L. et BERNAT DE CELIS, J. (1982), Peines perdues. Le système pénal en question, Paris, Le Centurion.

JACOUB, M. (1992), Les Inuit et la question pénale: le cas du Nouveau-Québec (titre provisoire), document de travail, thèse de doctorat en cours, École de criminologie, Université de Montréal.

KELSEN, H. (1953), Théorie pure du droit, Neuchâtel, Editions de la Baconnière, 1988.

KUHN, T. (1962), La structure des révolutions scientifiques, Paris, Flamarion, 1983.

LAPLANTE, J. (1992), « Le pénal et ses sujets : comment il les saisit. Y a-t-il une autre façon de voir et de faire?», département de criminologie, Université d'Ottawa.

LAPLANTE, J. (1991), «La question de la violence familiale et de son traitement », in L'intervention psychosociale au sein du pénal, Actes de la $1^{\text {èe }}$ Conférence internationale du GREPO, vol. 1, département de criminologie, Université d'Ottawa.

LAPLANTE, J. (1985), Crime et traitement, Montréal, Boréal Express.

LEMERT, E. (1951), Social Pathology, N.Y., McGraw-Hill Book Company, Inc.

LYNCH, M. J., GROVES, W. B. (1989), A Primer in Radical Criminology, N.Y., Harrow \& Heston.

LYOTARD, J.-F. (1979), La condition postmoderne, Paris, Éditions de Minuit.

MATZA, D. (1969), Becoming Deviant, New York, Prentice-Hall, Inc.

MEAD, G. H. (1917-18), * The Psychology of Punitive Justice », American Journal of Sociology, vol. $23, \mathrm{n}^{\circ} 5$, p. 577-602.

PARENT, C. (1992), «La contribution des études féministes sur la déviance des fernmes à la criminologie $»$, Criminologie, vol. $25, \mathrm{n}^{\circ} 2$, (dans ce numéro).

PARENT, C. (1991), Les féminismes et les paradigmes en criminologie, Université de Montréal, Ecole de criminologie, thèse de doctorat inédite.

PIRES, A. P. (1992), « La criminalité : enjeux épistémologiques, théoriques et pratiques» (titre provisoire), in F. Dumont, S. Langlois et Y. Martin (sous la direction de), Traité de pathologie sociale. Problèmes sociaux au Québec, (titre provisoire), Québec, Institut québécois de la recherche et de la culture.

PIRES, A. P. (1991), «Éthiques et réforme du droit criminel : au-delà des philosophies de la peine ", Ethica, vol. 3, n' 2, p. 47-78.

PIRES, A. P. (1990a), «Le devoir de punir: le rétributivisme face aux sanctions communautaires $»$, Revue canadienne de criminologie, vol. 32, $\mathrm{n}^{\circ} 3, \mathrm{p} .441-460$.

PIRES, A. P. (1990b), « La criminologie et ses objets paradoxaux: Réflexions épistémologiques sur un nouveau paradigme , Communication à la $1^{\text {tre }}$ journée du GCRN, Ecole de criminologie, Université de Montréal, le 30 novembre 1990 (a paraître). 
PIRES, A. P. et LANDREVILLE, P. (1985), «Les recherches sur les sentences et le culte de la loi », L'année sociologique, vol. 35, p. 83-113.

PIRES, A. P. (1983), Stigmate pénal et trajectoire sociale, Montréal, École de criminologie, Université de Montréal, thèse de doctorat inédite.

PIRES, A. P. (1979), "Le débat inachevé sur le crime: le cas du congrès de 1950 », Déviance et Société, vol. 3, $\mathrm{n}^{\circ} 1, \mathrm{p} .23-46$.

RAINS, P. (1975), «Imputations of Deviance: A Retrospective Essay on the Labeling Perspective», Social Problems, vol. 23, n 1, p. 1-11.

ROBERT, Ph. (1981), "De la «criminologie de la réaction sociale» à une sociologie pénale», L'Annéc sociologique, vol. 31, p. 253-283.

ROBERT, Ph. et KELLENS, G. (1973), «Nouvelles perspectives en sociologie de la déviance", Revue frangaise de sociologie, vol. 14, p. 371-395.

SACK. F. (1984), "Staat, Gesellschaft und politische Gewalt: Zur «Pathologie " politischer Konflikte», (p. 17-226) in F. Sack et H. Steinert (sous la direction de), Protest und Reaktion, Opladen, Westdeutscher Verlag.

SBRICCOLI, M. (1991), «Histoire de la criminalité et histoire du droit. Le rôle des sources juridiques dans l'histoire du crime et de la justice criminelle", $I \mathrm{AHCCl}$ Bulletin, vol. 14, p. 86-102.

SCHEERER, S. (1986), «Towards abolitionism », Contemporary Crisis, 10, p. 5-20.

SCHUTZ, A. (1987), Le chercheur et le quotidien, Paris, Méridiens Klincksieck.

SUTHERLAND, E. H. (1937), Le volem professionnel, Paris, Spress, 1962.

TAYLOR, I., WALTON, P., YOUNG, J. (1973), The New Criminology, London, Routledge \& Kegan Paul.

TOURAINE, A. (1984), Le retour de l'auteur, Paris, Fayard.

VEYNE, P. (1971), Comment on écrit l'histoire, Paris, Seuil, 1978.

WALGRAVE, L. (1986), " "La vulnérabilité sociétale: une tentative théorique pour comprendre la délinquance grave et persistante de certains jeunes ", in C. Detroy, F. Tulkens et M. van de Kerchove (sous la direction de), Délinquance des jeunes. Politiques et interventions, (p. 41-64), Bruxelles, E. Story-Scientia.

WACQUANT, L. J. D. (1992), «Introduction", in, P. Bourdieu et L. J. D. Wacquant, Réponses. Pour une anthropologie réflexive, Paris, Seuil. 